\section{Avaliação da aplicação da estratégia da
Atenção Integrada às Doenças Prevalentes \\ Avaliação da aplicação da estratégia da
Atenção Integrada às Doenças Prevalentes da Infância (AIDPI) por Agentes Comunitários de Saúde}

\section{Evaluation of the Integrated Management Childhood IIIness (IMCI) strategy application by Community Health Agents}

\begin{abstract}
Objectives: to assess the strategy for Integrated Management Childhood Illness by Community Health Agents.

Methods: assessment study accomplished in the cities of Recife and Olinda, Northeast Brazil. One hundred and ninety five structured questionnaires were applied by 106 community health agents during home visits to children under five years old in the period of October to November, 2002.

Results: $91,7 \%$ of the children under two months old were correctly assessed for signs of danger and 83,3\% for signs of alert. The assessment of conditions such as "nutrition", "growth" and "vaccination" indicated that the method was well performed in $96,9 \%$, $99 \%$ and $99 \%$ of the cases, respectively. In $73,1 \%$ of visits to children in the age group between two and five years old, the community health agents followed the protocol for danger signs correctly; as for alert signs the performance reached was of $80.8 \%$.

Conclusions: Community agents demonstrated they had the capability of learning and applying the strategy correctly. The "Procedure manual" demonstrated to be an adequate tool to address the health conditions of children under five years old in the community.
\end{abstract}

Key words Program evaluation, Child health, Child health service
Suely Arruda Vidal 1 Eroneide Valéria da Silva 2 Maria da Graça Oliveira 3 Ana Maria Siqueira 4 Eronildo Felisberto 5 Isabella Samico 6 Maria das Graças Santos Cavalcante 7

1-7 Instituto Materno Infantil de Pernambuco, IMIP. Diretoria de Pesquisas. Grupo de Estudos em Avaliação. Rua dos Coelhos, 300. Recife, PE, Brasil. CEP: 50.070-550. Tel: (81) 2122.4113

\section{Resumo}

Objetivos: avaliar a utilização da estratégia da Atenção Integrada às Doenças Prevalentes da Infância por Agentes Comunitários de Saúde.

Métodos: estudo do tipo avaliativo realizado nas cidades de Recife e Olinda, Nordeste do Brasil. Foram aplicados 195 questionários estruturados durante as visitas domiciliares realizadas por 106 agentes comunitários de saúde às crianças menores de cinco anos, no período de outubro a novembro de 2002.

Resultados: observou-se que 91,7\% dos menores de dois meses foram avaliados corretamente quanto aos sinais de perigo e 83,3\% quanto aos sinais de alerta. A avaliação das seções sobre "alimentação", "crescimento" e "vacina" demonstrou que o método foi bem aplicado em 96,9\%, 99\% e 99\% dos casos, respectivamente. Em 73,1\% das visitas às crianças na faixa etária entre dois meses e cinco anos, os agentes de saúde seguiram corretamente a padronização para verificação dos sinais de perigo e em $80,8 \%$ para os sinais de alerta.

Conclusões: verificou-se que os agentes comunitários foram capazes de apreender o conteúdo e aplicar corretamente a estratégia. $O$ "Manual de condutas" demonstrou ser um adequado instrumento à correta abordagem dos problemas de saúde dos menores de cinco anos na comunidade.

Palavras-chave Avaliação de programas, Saúde infantil, Serviços de saúde infantil 


\section{Introdução}

A Reunião de Alma Ata, em 1978, pode ser consi-derada como o marco histórico da Atenção Primária em Saúde (APS) definida como a chave para obtenção de níveis de saúde que permitissem as populações levar uma vida socialmente e economicamente produtiva. ${ }^{1}$ Starfield 2 define a APS como a porta de entrada no sistema de saúde, fornecendo atenção sobre a pessoa e devendo ser o eixo orientador determinante do trabalho dos demais níveis hierárquicos desse sistema, cuja diretriz é a promoção, a manutenção e a melhoria da saúde. Os principais componentes da APS são: educação em saúde, saneamento básico e ambiental, programas de saúde materno-infantil, prevenção e tratamento de doenças endêmicas e comuns, medicamentos essenciais e promoção da nutrição. ${ }^{1-3}$

Com base nestes referenciais, o Instituto Materno Infantil de Pernambuco (IMIP), em Recife, criou, em 1983,4 o Projeto de Extensão Comunitária, apoiado pelo Fundo das Nações Unidas para a Infância (UNICEF), cujo objetivo era desenvolver um modelo de atenção primária no nível comunitário, que permitisse acesso eqüitativo e fornecesse cobertura universal ao grupo materno-infantil. Baseava-se no trabalho de Agentes Comunitários de Saúde (ACS), de pediatras e de ginecologistas capacitados para desenvolverem ações básicas e educação em saúde para este grupo específico, com metodologia de trabalho apoiada em visitas domiciliares e na unidade de saúde local, fortalecendo a ligação entre o serviço e a comunidade.

Esse projeto foi um precursor do Programa de Agentes Comunitários de Saúde (PACS), concebido em 1991 pelo Ministério da Saúde do Brasil, ano do inicio da sua implantação em escala nacional. ${ }^{5}$

Em sequência ao PACS, surgiu em 1994, o Programa Saúde da Família (PSF), como um avanço para o cumprimento dos princípios de universalidade, eqüidade e integralidade do Sistema Único de Saúde (SUS). O PSF constitui-se em uma estratégia para mudança do modelo assistencial baseada nos pressupostos da atenção primária. Tem como foco de atenção a família, entendida a partir do meio onde vive, atuando na prestação de assistência integral, na vigilância aos grupos de risco, na prevenção, recuperação e promoção da saúde. 6

Neste contexto, o PSF se insere como mais um recurso para o alcance das metas estabelecidas no "Pacto pela Infância" e, dentro deste propósito, o Ministério da Saúde do Brasil adotou em 1995, como política de atenção à saúde da criança menor de cinco anos, a estratégia da Atenção Integrada às
Doenças Prevalentes da Infância (AIDPI), elaborada pela Organização Mundial da Saúde (OMS) Organização Pan-Americana da Saúde (OPAS) e o UNICEF. 7-10

A estratégia AIDPI incorporou as ações do Programa de Assistência Integral à Saúde da Criança (PAISC), porém introduzindo o conceito de integralidade. Propõe um novo modelo de abordagem à saúde da criança no primeiro nível de atenção, sistematizando o atendimento clínico e integrando ações curativas com medidas preventivas e de promoção da saúde. ${ }^{7-10}$

A partir de 1996 começaram a ser treinados, no Brasil, profissionais médicos e enfermeiros para operacionalização da AIDPI. Contudo, para a efetiva implantação da estratégia e consequente melhoria da qualidade da atenção à saúde da criança dentro da perspectiva do novo modelo assistencial, fazia-se necessário o treinamento de todos os profissionais das equipes, inclusive dos agentes comunitários de saúde. Nesse novo modelo assistencial, onde o PSF é a porta de entrada para o sistema, a capacitação dos agentes amplia o acesso principalmente da população infantil.

Para isso, o material instrucional foi adaptado por técnicos do Ministério da Saúde e do IMIP, sendo lançadas duas publicações, o "Manual de condutas para Agentes Comunitários de Saúde: Atenção Integrada às Doenças Prevalentes na Infância (AIDPI)" e "Atenção básica à saúde da criança: texto de apoio para Agentes Comunitários de Saúde."11,12

Alguns estudos têm abordado o processo de implantação da estratégia AIDPI para profissionais de nível superior, e dentro deste, a sua aplicação.13,14 Para Agentes Comunitários de Saúde entretanto, o presente trabalho é uma investigação pioneira no Brasil. É importante realizar, neste momento, uma avaliação do processo em virtude de: 1) a política do Ministério da Saúde para a área técnica de saúde da criança estar direcionada à implementação da estratégia AIDPI 15 e 2) por estar contida na proposta do Programa de Expansão e Consolidação do Saúde da Família (PROESF), cujos objetivos são: reorganizar a atenção básica (conversão do modelo assistencial), qualificar os recursos humanos e reforçar os sistemas de informação para avaliação dos serviços de atenção básica. ${ }^{16}$

Assim este trabalho tem por objetivo avaliar a aplicação da estratégia AIDPI por agentes comunitários de saúde mediante a observação do uso do "Manual de condutas" durante as visitas domiciliares às crianças menores de cinco anos pelos referidos ACS. 


\section{Métodos}

Trata-se de um estudo avaliativo normativo, com abordagem do processo realizado nos meses de outubro a novembro de 2002, dois meses após a capacitação de 106 ACS de 19 equipes de Saúde da Família, lotadas em 10 Unidades de Saúde, de cinco comunidades das cidades de Recife e de Olinda, Nordeste do Brasil.
O estudo desenvolveu-se na área de abrangência das comunidades dos PSF, onde os ACS foram acompanhados durante as visitas domiciliares por supervisores, pediatras preceptores ou enfermeiros das unidades às quais estão vinculados.

Neste acompanhamento os supervisores, munidos de dois instrumentos de avaliação - um para bebês menores de dois meses e o outro para crianças de dois meses a cinco anos, observavam o manuseio

Figura 1

Fluxograma para avaliação de crianças menores de cinco anos pelos Agentes Comunitários de Saúde (ACS) na estratégia Atenção Integrada às Doenças Prevalentes na Infância (AIDPI).

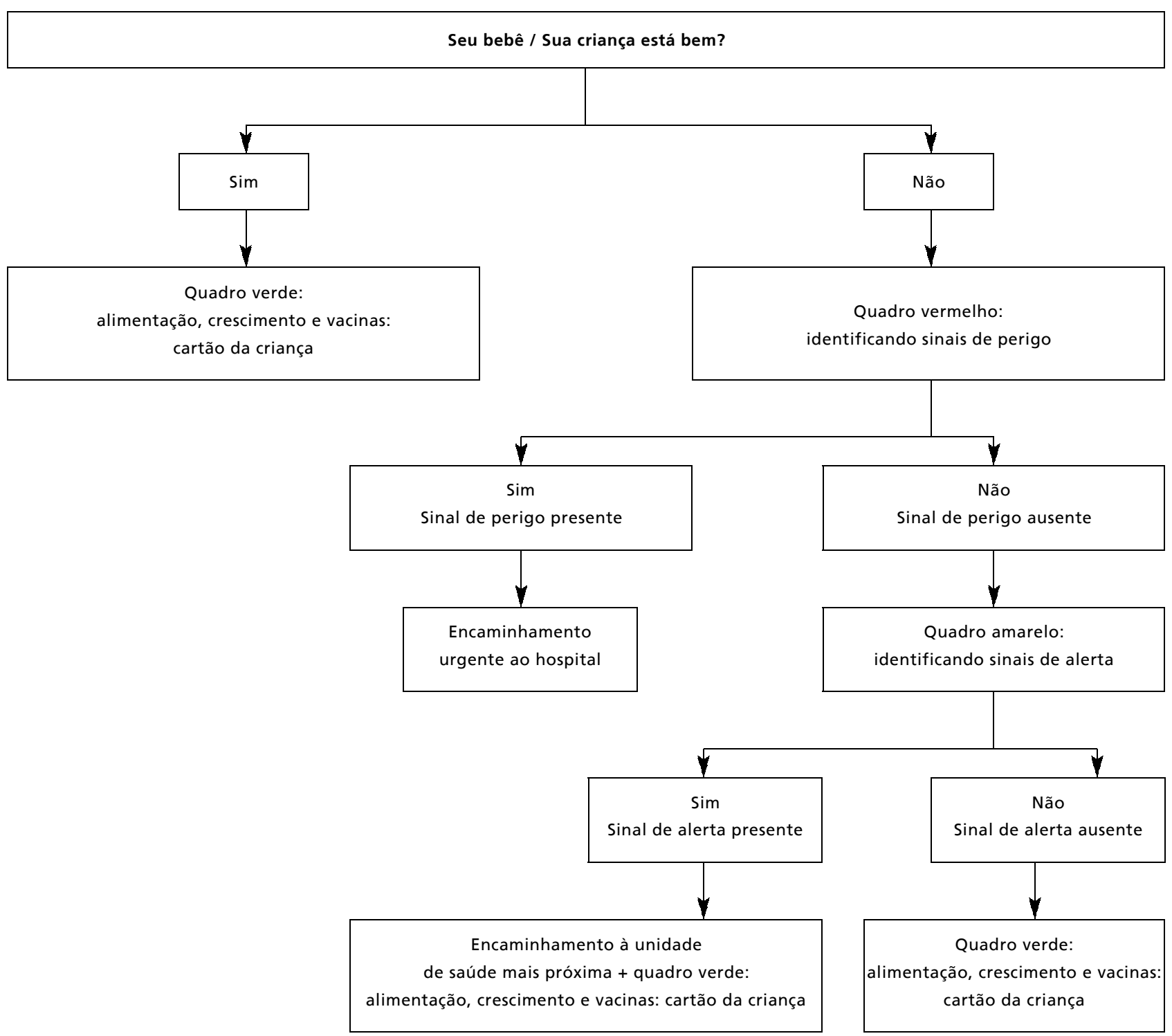

Fonte: Ministério da Saúde. Atenção básica à saúde na infância. Brasília (DF): 2001.12 
do "Manual de condutas" e a aplicação da estratégia pelos ACS, anotando sem interferir, as observações. Ao final, se detectada alguma dificuldade ou incorreção, estas eram corrigidas. Foram aplicados 195 questionários durante as visitas realizadas no período acima indicado, os quais foram utilizados neste estudo.

As perguntas contidas nos instrumentos eram fechadas, abordavam aspectos relativos ao estado de saúde da criança (presença de sinais clínicos) e encaminhamentos necessários para a resolução do problema, questões referentes à prevenção de doenças (vacinas) e à promoção da saúde (aleitamento materno, alimentação e cuidados de saúde) e seguiam a mesma ordem dos passos sistematizados pela AIDPI. ${ }^{9}$ A Figura 1 apresenta a sequência da avaliação da criança preconizada pelo "Manual de condutas". 11

Para análise dos dados foi utilizado o programa de informática Epi-info, versão 6.0 e os resultados estão apresentados em tabelas de distribuição de frequência.

\section{Resultados}

Conforme o indicado na Tabela 1, 97 crianças eram menores de dois meses a cinco anos incompletos. Verifica-se também que em apenas $8,2 \%$ dos menores de cinco anos avaliados, a sequência pre- conizada pela AIDPI não foi utilizada corretamente. O percentual de $12,3 \%$ rotulado como "não se aplica" se refere aos casos onde as mães/responsáveis relataram que as crianças não estavam bem. Estas foram então avaliadas quanto aos sinais de perigo e de alerta, específicos para cada faixa etária.

A Tabela 2 apresenta a sequência sistematizada pelo "Manual de condutas para avaliação dos menores de dois meses". Do total de 12 bebês com relato sobre algum problema de saúde, 11 foram avaliados corretamente quanto aos sinais de perigo, e destes, um foi encaminhado com urgência ao hospital. Verifica-se que os sinais de alerta foram bem avaliados em $83,3 \%$ dos casos.

Ainda na Tabela 2 se observa que a seção "Avaliação da amamentação do bebê, do crescimento e da situação vacinal" foi bem aplicada na grande maioria das vezes $(96,9 \%, 99,0 \%$ e $99,0 \%$, respectivamente). Apenas em um caso o agente de saúde não soube preencher o gráfico do peso e em outro a situação vacinal não foi verificada.

$\mathrm{Na}$ Tabela 3 verifica-se que as orientações fornecidas pelos ACS para amamentação e para os cuidados com o bebê seguiram a descrição do "Manual do AIDPI" em 96,9\% e 80,4\% dos casos (respectivamente). Quanto às visitas de retorno realizadas para os problemas de amamentação, 20\% não seguiram a norma escrita para as orientações, contudo observouse melhora quanto às recomendações para bebês sadios, havendo apenas $4 \%$ de discordância.

Tabela 1

Número de visitas realizadas pelos Agentes Comunitários de Saúde (ACS) às crianças segundo faixa etária e uso correto da seqüência para "criança está bem" do "Manual de condutas da estratégia AIDPI". Instituto Materno Infantil de Pernambuco (IMIP), Recife, PE, 2002.

\begin{tabular}{|c|c|c|c|c|c|c|}
\hline \multirow{3}{*}{ Uso correto da seqüência } & \multicolumn{4}{|c|}{ Faixa etária } & \multirow{2}{*}{\multicolumn{2}{|c|}{ Total }} \\
\hline & \multicolumn{2}{|c|}{$<2$ meses } & \multicolumn{2}{|c|}{2 meses a 5 meses } & & \\
\hline & $\mathrm{n}$ & $\%$ & $\mathrm{n}$ & $\%$ & $\mathrm{n}$ & $\%$ \\
\hline Não & 5 & 5,2 & 11 & 11,2 & 15 & 8,2 \\
\hline Não se aplica* & 11 & 11,3 & 13 & 13,3 & 24 & 12,3 \\
\hline Total de visitas & 97 & 100,0 & 98 & 100,0 & 195 & 100,0 \\
\hline
\end{tabular}

* Casos em que as mães relataram que a criança não estava bem 
Distribuição das visitas realizadas pelos Agentes Comunitários de Saúde (ACS) utilizando a estratégia Atenção Integrada às Doenças Prevalentes da Infância (AIDPI), segundo uso correto das principais seções do "Manual de condutas... para bebês menores de dois meses". Instituto Materno Infantil de Pernambuco (IMIP), Recife, PE, 2002.

\begin{tabular}{|c|c|c|c|c|c|c|}
\hline \multirow{2}{*}{ Avaliação correta das seções } & \multicolumn{2}{|c|}{ Sim } & \multicolumn{2}{|c|}{ Não } & \multicolumn{2}{|c|}{ Total } \\
\hline & $\mathrm{n}$ & $\%$ & $\mathrm{n}$ & $\%$ & $\mathrm{n}$ & $\%$ \\
\hline Identificando sinais de perigo & 11 & 91,7 & 1 & 8,3 & 12 & 100,0 \\
\hline Protegendo o bebê a caminho do hopital & 1 & 100,0 & 0 & 0,0 & 1 & 100,0 \\
\hline Identificando sinais de alerta & 10 & 83,3 & 2 & 16,7 & 12 & 100,0 \\
\hline Procurando ajuda de imediato & 0 & 0,0 & 1 & 100,0 & 1 & 100,0 \\
\hline Visita domiciliar após retorno da Unidade de Saúde & 2 & 100,0 & 0 & 0,0 & 2 & 100,0 \\
\hline Avaliação da amamentação & 94 & 96,9 & 3 & 3,1 & 97 & 100,0 \\
\hline Avaliação do crescimento & 96 & 99,0 & 1 & 1,0 & 97 & 100,0 \\
\hline Avaliação da Situação Vacinal & 96 & 99,0 & 1 & 1,0 & 97 & 100,0 \\
\hline
\end{tabular}

Nota: Cinco das seções têm totais diferentes de 97 porque estas não se aplicam a todas as crianças.

Tabela 3

Distribuição das visitas realizadas pelos Agentes Comunitários de Saúde (ACS) aos menores de dois meses utilizando a estratégia Atenção Integrada às Doenças Prevalentes da Infância (AIDPI), segundo realização correta dos itens sitematizados nas seções consideradas do "Manual de condutas". Instituto Materno Infantil de Pernambuco (IMIP), Recife, PE, 2002.

\begin{tabular}{|c|c|c|c|c|c|c|}
\hline \multirow{2}{*}{ Realização correta das seções } & \multicolumn{2}{|c|}{ Sim } & \multicolumn{2}{|c|}{ Não } & \multicolumn{2}{|c|}{ Total } \\
\hline & $\mathrm{n}$ & $\%$ & $\mathrm{n}$ & $\%$ & $\mathrm{n}$ & $\%$ \\
\hline Orientando amamentação e seus problemas & 94 & 96,9 & 3 & 3,1 & 97 & 100,0 \\
\hline Visita retorno para problemas de alimentação & 16 & 80,0 & 4 & 20,0 & 20 & 100,0 \\
\hline Visita de retorno para bebê sadio & 73 & 96,1 & 3 & 3,9 & 76 & 100,0 \\
\hline Cuidando do bebê & 78 & 80,4 & 19 & 19,6 & 97 & 100,0 \\
\hline
\end{tabular}

Nota: Duas das seções têm um total diferente de 97 porque estas não se aplicam a todas as crianças.

As observações relativas às crianças na faixa etária de dois meses a cinco anos encontram-se na Tabela 4. Das 98 crianças visitadas, 26 apresentaram algum problema de saúde, necessitando avaliação quanto aos sinais de perigo e de alerta. Os sinais de perigo foram avaliados conforme a padronização em $73,1 \%$ dos casos e os sinais de alerta em $80,8 \%$. As seções: "Fique atento" e "Ensinando mães ou responsáveis a administrar medicação" foram aplicadas adequadamente em $78,3 \%$ e $78,9 \%$ das visitas, respectivamente. Ainda nesta tabela verifica-se o percentual de acertos quanto à avaliação preconizada para ori- entação sobre alimentação $(87,8 \%)$, crescimento $(96,9 \%)$ e vacinas $(99 \%)$.

Quanto ao uso de alguns instrumentos preconizados pelo PSF e pela AIDPI para o acompanhamento de crianças menores de cinco anos, verificou-se que em $91,8 \%$ das visitas o ACS conduzia a "Folha de registro", porém, apenas $19 \%$ dispunham da cópia do "Cartão da criança." Por outro lado, em 100\% das visitas os ACS portavam o "Manual de condutas da AIDPI" (Tabela 5) e 91\% demonstraram saber manuseá-lo corretamente. 
Distribuição das visitas realizadas pelos Agentes Comunitários de Saúde (ACS) às crianças na faixa etária de dois meses a cinco anos utilizando a estratégia Atenção Integrada às Doenças Prevalentes da Infância (AIDPI), segundo uso correto de algumas seções do "Manual de condutas". Instituto Materno Infantil de Pernambuco (IMIP), Recife, PE, 2002.

\begin{tabular}{|c|c|c|c|c|c|c|}
\hline \multirow{2}{*}{ Avaliação correta das seções } & \multicolumn{2}{|c|}{ Sim } & \multicolumn{2}{|c|}{ Não } & \multicolumn{2}{|c|}{ Total } \\
\hline & $\mathrm{n}$ & $\%$ & $\mathrm{n}$ & $\%$ & $\mathrm{n}$ & $\%$ \\
\hline Identificando sinais de perigo & 19 & 73,1 & 7 & 26,9 & 26 & 100,0 \\
\hline Identificando sinais de alerta & 21 & 80,8 & 5 & 19,2 & 26 & 100,0 \\
\hline Visita domiciliar após retorno da Unidade de Saúde & 3 & 37,5 & 5 & 62,5 & 8 & 100,0 \\
\hline Fique atento & 18 & 78,3 & 5 & 5,1 & 23 & 100,0 \\
\hline Ensinando mães a administrar a medicação & 15 & 78,9 & 4 & 21,1 & 19 & 100,0 \\
\hline Orientação sobre alimentação & 86 & 87,8 & 12 & 12,2 & 98 & 100,0 \\
\hline Orientação sobre vacinas & 97 & 99,0 & 1 & 1,0 & 98 & 100,0 \\
\hline Orientação sobre crescimento & 95 & 96,9 & 3 & 3,1 & 98 & 100,0 \\
\hline
\end{tabular}

Nota: As cinco primeiras seções têm totais diferentes de 98 porque estas não se aplicam a todas as crianças.

Tabela 5

Distribuição das visitas realizadas pelos Agentes Comunitários de Saúde (ACS) aos menores de cinco anos utilizando a estratégia Atenção Integrada às Doenças Prevalentes da Infância (AIDPI) segundo uso de instrumentos preconizados pelo Programa Saúde da Família (PSF) e a estratégia Atenção às Doenças Prevalentes da Infância (AIDPI). Instituto Materno Infantil de Pernambuco (IMIP), Recife, PE, 2002.

\begin{tabular}{|c|c|c|c|c|c|c|}
\hline \multirow{2}{*}{$\begin{array}{l}\text { Instrumentos usados pelos } \\
\text { Agentes Comunitários de Saúde }\end{array}$} & \multicolumn{2}{|c|}{ Sim } & \multicolumn{2}{|c|}{ Não } & \multicolumn{2}{|c|}{ Total } \\
\hline & $\mathrm{n}$ & $\%$ & $\mathrm{n}$ & $\%$ & $\mathrm{n}$ & $\%$ \\
\hline Folha de registro & 179 & 91,8 & 16 & 8,2 & 195 & 100,0 \\
\hline Cartão da criança (cópia) & 37 & 19,0 & 158 & 81,0 & 195 & 100,0 \\
\hline Manual de condutas & 195 & 100,0 & 0 & 0,0 & 195 & 100,0 \\
\hline
\end{tabular}

\section{Discussão}

Neste estudo ficou evidenciado que os ACS se mostraram aptos a seguir os passos preconizados pela estratégia AIDPI para identificação dos sinais de perigo e de alerta em mais de $80 \%$ dos bebês menores de dois meses e mais de $70 \%$ das crianças de dois meses a cinco anos. Esta competência aumenta, portanto, a possibilidade de intervenção precoce, reduzindo o risco de complicações e, conseqüentemente, contribuindo para a redução da mortalidade, como relata Benguigui. ${ }^{17}$ Assim, alguns problemas ainda não percebidos ou valorizados pela família podem ser detectados durante as visitas domiciliares e encaminhados aos serviços de saúde para o tratamento oportuno. 17

Outro aspecto positivo da aplicação da AIDPI pelos ACS é a integração da abordagem dos problemas de saúde mais frequentes nas crianças menores de cinco anos com as ações de promoção e de prevenção, por avaliar sistematicamente o estado nutricional da criança, a alimentação e as vacinas. Estas recomendações, difundidas na comunidade, contribuem para modificar atitudes e práticas de pais ou 
responsáveis, tornando-os mais participativos nos cuidados com a criança para que ela cresça de forma mais saudável.17

Quanto a esses aspectos os resultados obtidos no presente trabalho indicam altos percentuais de aplicação correta (mais de $85 \%$ para ambos grupos etários) demonstrando melhor desempenho do que na sequência relativa à avaliação dos sinais. Estes achados podem ser justificados por fazerem parte de um conhecimento adquirido previamente à capacitação na AIDPI e já incorporado às atividades desenvolvidas na comunidade, como tem sido observado em outros estudos. 18-20

Além destas vantagens, o seguimento domiciliar pós-consulta na unidade de saúde, deveria ser realizado via de regra para ensino e supervisão da administração de medicamento, eliminando dúvidas e contribuindo para uma maior aderência ao tratamento. No tocante a estes itens, porém, o número de visitas foi muito pequeno neste trabalho, não permitindo uma melhor análise.

Várias experiências a favor de programas de atenção primária em saúde baseada em Agentes Comunitários foram relatadas, como a do Nepal em 1994, que implementou a cobertura vacinal, o uso de sais de rehidratação oral, o acompanhamento do crescimento, a adesão ao tratamento das helmintíases, assim como as atividades educativas para saúde, dentro de um programa de educação nutricional para redução da hipovitaminose A. No entanto, o sucesso desse programa deveu-se, segundo os autores, ao treinamento, à supervisão, ao fornecimento dos suprimentos e à associação de atividades curativas às de prevenção e promoção da saúde. 18

Resultados favoráveis ao trabalho dos ACS na vigilância às crianças de risco e o emprego de ações básicas de saúde também foram encontrados em dois municípios da Zona da Mata de Pernambuco em 2000, contribuindo para a redução da mortalidade infantil. 19

Ainda no Nordeste do Brasil o estudo de Svitone et al. ${ }^{21}$ demonstrou redução dos níveis de desnutrição e queda na taxa de mortalidade infantil, relacionados ao trabalho desenvolvido por agentes comunitários de saúde.

Em estudo realizado no Sudeste do Brasil, em um dos onze municípios do estado de São Paulo com piores índices de desenvolvimento infantil, César et al.,22 em 2002, relataram que aqueles profissionais, bem capacitados, podem contribuir para a melhoria dos indicadores, reduzindo assim taxa de hospitalização e aumentando a utilização do soro de rehidratação oral e a cobertura vacinal.

Entretanto, Berman et al.,20 em estudo de re- visão, mencionam a dificuldade de avaliar tais programas, pois isto depende dos objetivos propostos, os quais são variáveis. Referem esses autores que algumas avaliações podem indicar resultados falso positivos, por não considerarem a influência de cointervenções, como também falso negativos, pelo curto espaço de tempo para obtenção de resultados, principalmente em locais com péssimas condições socioeconômicas e ambientais precárias. Concluíram que para os serviços de atenção primária baseados em Agentes Comunitários tornarem-se efetivos e produzirem impacto significante nos indicadores de saúde, são necessários maiores investimentos em treinamento, supervisão e apoio logístico.

A implantação do PSF no Brasil possibilita a superação destas dificuldades por proporcionar apoio logístico, supervisão diária, treinamento em serviço e por aumentar a resolutividade dos problemas de saúde ao fixar uma equipe de saúde na comunidade a ser assistida.

Os agentes de saúde são pessoas residentes nas comunidades onde o PACS ou PSF está implantado, com baixo nível de instrução formal e tendo recebido poucas semanas de treinamento inicial direcionado às tarefas padronizadas a serem executadas. São supervisionados por enfermeiros, devendo receber instruções adicionais a partir de um programa de educação continuada, o que nem sempre acontece.

Segundo Nunes et al. 23 os ACS, por fazerem parte da comunidade, servem de tradutores bi-direcionais dos saberes do processo saúde-doença de um lado, e de outro, das necessidades da população, sentindo-se orgulhosos de dominarem certos conteúdos biomédicos que os diferenciam na comunidade (algumas vezes, no entanto pouco satisfeitos pela insuficiente capacitação que recebem). Este sentimento de "orgulho" muito provavelmente aumenta a motivação para a rotina do trabalho e a integração da equipe do PSF.

Por ser direcionada às doenças prevalentes da infância, esta estratégia encontra grande receptividade entre todos os profissionais, porque além da facilidade constatada no manuseio do "Manual de condutas", atende e responde às queixas mais frequentes das mães/responsáveis pelas crianças.

Estudo realizado em Pernambuco, evidenciou a receptividade e a adesão dos profissionais de nível superior do PSF à estratégia a partir do relato de todos os entrevistados, os quais foram unânimes ao referirem que a AIDPI oferece maior segurança e qualidade no atendimento às crianças. 13

A estratégia cresce de importância e amplia seu alcance se utilizada pelos ACS, tendo em vista a rotina de seu trabalho, ao visitar diariamente os lares de 
crianças menores de cinco anos, desde os primeiros dias de vida, sendo de fato, o primeiro contato daqueles indivíduos com o sistema de saúde.

Para maior adesão à estratégia faz-se necessário entretanto o envolvimento de todos os profissionais da equipe. Segundo Rowe et al. ${ }^{24}$ a presença de intervenções simultâneas no sentido de fornecer um melhor suporte aos profissionais, não limitadas apenas ao treinamento, promoveria, de forma mais consistente, uma melhor atuação tal como apoio à supervisão e à valorização profissional.

Conclui-se que a aplicação da estratégia AIDPI pelos ACS possibilita um trabalho em sinergia com a equipe de profissionais da unidade de saúde, apoiando e fortalecendo as ações implementadas, especialmente aquelas consideradas para o componente comunitário da estratégia. 10,25 Em atuação mais integrada é possível minimizar os graves problemas que afetam a saúde das rianças de modo que possam desenvolver-se como pessoas mais saudáveis. Conclui-se ainda que estes profissionais, mesmo possuindo baixo nível de escolaridade, são capazes de apreender o contéudo da estratégia e aplicá-la adequadamente nas comunidades.

\section{Referências}

1. OMS (Organización Mundial de la Salud). Atención primária de salud. In: Conferencia Internacional sobre Atención Primaria de Salud; 1978 Set 6-12; Alma-Ata, URSS. Ginebra: A Organización; 1978.

2. Starfield, B. Atenção primária: equilíbrio entre necessidades de saúde, serviços e tecnologia. Brasília (DF): Ministério da Saúde; 2002.

3. Ebrahim GJ. Primary health care in 21 st century. Rev Bras Saude Matern Infant 2001; 1: 199-201.

4. Mendonça LC, Mendonça JH. IMIP: identidade, missão e trajetória. Recife: Bagaço; 2000.

5. Ministério da Saúde, Fundação Nacional de Saúde. Programa de agentes comunitários de saúde: avaliação qualitativa do PACS. Brasília (DF): O Ministério; 1994.

6. Ministério da Saúde. Saúde da família: uma estratégia para reorientação do modelo assistencial. Brasília (DF): O Ministério; 1997.

7. Ministério da Saúde. Metas da Cúpula Mundial em Favor da Infância: avaliação de meia década, 1990-1995. Brasília (DF): O Ministério; 1997.

8. Benguigui Y, Land S, Paganini JM, Yunes J, editores. Ações de saúde materno infantil a nível local segundo as Metas da Cúpula Mundial em Favor da Infância. Washington (DC): OPAS; 1997. (Serie HCT/AIEPI-4)

9. Ministério da Saúde, OMS (Organização Mundial de Saúde), OPAS (Organização Pan-Americana de Saúde). Atenção integrada às doenças prevalentes da infância: curso de capacitação. Brasília (DF): O Ministério; 1998.

10. Benguigui Y. Perspectivas em el control de enfermedades em los niños: Atención Integrada a las Enfermedades Prevalentes de la Infância. Rev Bras Saúde Materno Infantil 2001; 1: 7-19.

11. Ministério da Saúde. Manual de condutas para Agentes Comunitários de Saúde: Atenção Integrada às Doenças Prevalentes na Infância - AIDPI: procedimentos para bebês menores de 2 meses e procedimentos para crianças de 2 meses a 5 anos. Brasília (DF): O Ministério; 2001.

12. Ministério da Saúde. Atenção básica à saúde da criança: texto de apoio para o agente comunitário de saúde; Atenção integrada às doenças prevalentes na infância Aidpi. Brasília (DF): O Ministério; 2001.

13. Felisberto E, Samico I, Carvalho EF, Maggi, RS. Avaliação do processo de implantação da estratégia da Atenção Integrada às Doenças Prevalentes da Infância no Programa de Saúde da Família, no estado de Pernambuco, Brasil. Cad Saúde Pública 2002; 18: 1737-45.

14. Samico I. Avaliação da atenção à saúde da criança: um estudo de caso no estado de Pernambuco [tese doutorado]. Recife: Escola Nacional de Saúde Pública da Fundação Oswaldo Cruz; 2003.

15. Ministério da Saúde. Secretaria de Políticas de Saúde. Brasília (DF): O Ministério; 2001. (Série C. Projetos, Programas e Relatórios, n. 50).

16. Ministério da Saúde. Projeto de expansão e consolidação do saúde da família (PROESF): Acordo de empréstimo n. ${ }^{\circ}$ 7105-BR. Brasília (DF): O Ministério; 2003. Disponível em: http://dtr2002.saude.gov.br/proesf/14material/apresentacao/apres3.pdf. Acesso em: [2003 maio 2].

17. Benguigui Y. Integrated Managemnet of Childhood Illness (IMCI): na innovative vision for child health care. Rev Bras Saúde Matern Infant 2001; 1: 223-36.

18. Curtalo F, Siwakoti B, Lagrosa C, LaRaja M, Guerra R. Improving skills and utilization of community health volunteers in Nepal. Soc Sci Med 1995; 40: 1117-25.

19. Burmaz T. Avaliação da influência do Projeto Salva-Vidas sobre as atividades dos agentes comunitários de saúde em dois municípios da Zona da Mata Pernambucana, 1998/99 [dissertação mestrado]. Recife: Instituto Materno Infantil de Pernambuco, IMIP; 2000.

20. Berman PA, Gwatkin DR, Burgr SE. Community-based health workers; head start or false start towards health for all? Sc Sci med 1987; 25: 443-59.

21. Svitone EC, Garfield, R, Vasconcelos MI, Craveiro VA. Primary health care lessons from the Northeast of Brazil: the Agentes de Saúde Program. Rev Panam Salud Publica 2000; 7: 293-302.

22. César JÁ, Cavaleti MA, Holthausen RS, Lima LGS. Mudanças em indicadores de saúde em um município com agentes comunitários: o caso de Itapirapuã Paulista, Vale do Ribeira, São Paulo, Brasil. Cad Saúde Pública 2002; 18 1647-54.

23. Nunes MO, Trad LD, Almeida BA, Homem CR, Melo MCIC. O agente comunitário de saúde: construção da 
identidade desse personagem híbrido e polifônico. Cad Saúde Pública 2002; 18: 1639-46.

24. Rowe AK, Onikpo F, Lama M, Cokou F, Deming MS. Management of childhood illness at health facilities in Benin: problems and their causes. Am J Public Health 2001; 91: 1625-35.
25. OPS (Organización Panamericana de la Salud), OMS (Organización Mundial de la Salud). Atención Integrada a las Enfermedades Prevalentes de la Infancia: informe AIEPI del programa global OMS - 1996-1997. Washington (DC): Las Organizaciones; 1999. (Serie HCP/HCT/AIEPI-99.2).

Recebido em 3 de janeiro de 2003

Versão final reaparesentada em 7 de abril de 2003

Aprovado em 5 de maio de 2003 